Georgia State University

ScholarWorks @ Georgia State University

\title{
Attention Bias in Adult Survivors of Childhood Maltreatment with and without Posttraumatic Stress Disorder
}

Negar Fani

Georgia State University, nfani@emory.edu

Bekh Bradley-Davino

Emory University, rbradl2@emory.edu

Kerry J. Ressler

Emory University, kressle@emory.edu

Erin B. McClure-Tone

Georgia State University, etone@gsu.edu

Follow this and additional works at: https://scholarworks.gsu.edu/psych_facpub

Part of the Psychology Commons

\section{Recommended Citation}

Fani, Negar; Bradley-Davino, Bekh; Ressler, Kerry J.; and McClure-Tone, Erin B., "Attention Bias in Adult Survivors of Childhood Maltreatment with and without Posttraumatic Stress Disorder" (2011). Psychology Faculty Publications. 125.

https://scholarworks.gsu.edu/psych_facpub/125

This Article is brought to you for free and open access by the Department of Psychology at ScholarWorks @ Georgia State University. It has been accepted for inclusion in Psychology Faculty Publications by an authorized administrator of ScholarWorks @ Georgia State University. For more information, please contact scholarworks@gsu.edu. 
Attention Bias in Adult Survivors of Childhood Maltreatment with and without Posttraumatic Stress Disorder

Negar Fani' ${ }^{1}$, Bekh Bradley-Davino ${ }^{2,3}$, Kerry J. Ressler², Erin B. McClure-Tone, Ph.D. ${ }^{1}$

1 Department of Psychology, Georgia State University, Atlanta, GA

2 Department of Psychiatry and Behavioral Sciences, Emory University, Atlanta, GA

3 Atlanta VA Medical Center

Address correspondence to:

Negar Fani, M.S.

Department of Psychology

Georgia State University

140 Decatur Street

Suite 774

Atlanta, GA 30303-3083

Phone: (404) 413-6341

Fax: (404) 413-6218

Email: nfani1@student.gsu.edu

Key words: Posttraumatic Stress Disorder, Emotion processing, Childhood maltreatment, Dot Probe, Attention bias 
Reports of maltreatment in early childhood, in the forms of physical, sexual, or emotional abuse, have become increasingly common (U.S. Department of Health and Human Services, 1996). Childhood maltreatment is associated with a variety of negative outcomes, including drug and alcohol abuse, physical health problems, and risky sexual behavior (Repetti, Taylor \& Seeman, 2002). Children who have experienced early life trauma also demonstrate greater vulnerability than non-traumatized peers to Posttraumatic Stress Disorder (PTSD), both in the immediate aftermath of childhood trauma and in the context of adverse events that occur later in development (Bremner et al., 1993; Golier et al., 2003; Widom, 1999). However, only approximately a third of individuals who have experienced childhood maltreatment develop PTSD in their lifetimes (Widom, 1999). Characteristics that elevate risk for PTSD in this subgroup of maltreated youths remain unclear; cognitive features, such as maladaptive information processing styles, constitute one potential set of risk-related factors.

Neuropsychological findings suggest that adults who have endured trauma and developed PTSD demonstrate biases in attention, cue interpretation, and memory when compared with trauma survivors who did not develop PTSD (for a review, see Vasterling \& Brailey, 2005). Emotion processing theorists, such as Foa and Kozak (1986), postulate that, after trauma, some individuals develop pathological cognitive frameworks, or “fear structures," that lead them to perceive mildly threatening or ostensibly benign stimuli (e.g., visual cues that vaguely resemble the original trauma stimulus) as threatening. They then are prone to respond both behaviorally and cognitively to such stimuli in exaggerated ways. Visual cues appear to be especially salient triggers for individuals with PTSD (Foa \& Kozak, 1986); therefore, biased attention to and processing of incoming visual information may be particularly relevant to post-traumatic psychopathology. In particular, it appears that trauma survivors with PTSD demonstrate 
attentional biases to stimuli that are relevant to their own traumatic experiences (for a review, see Buckley, Blanchard \& Neill, 2000). While these selective attentional patterns may be adaptive in the immediate presence of threat, their persistence after genuine threats have subsided can disrupt downstream information processing and perpetuate anxious symptoms.

Researchers have typically used one of two cognitive paradigms to measure biases in attention to threat in individuals with PTSD: the emotional Stroop task and the dot probe or visual probe task. Both paradigms require individuals to filter out interfering emotional cues in order to perform non-emotional attention tasks. Emotional Stroop paradigms involve naming the colors in which trauma-related and benign words are printed; studies using such measures to examine attentional bias in individuals with PTSD have found evidence of extended response latencies to threat-related words in veteran populations (McNally et al., 1990; McNally, English \& Lipke, 1993; Kaspi, McNally \& Amir, 1995; Vrana, Roodman \& Beckham, 1995), rape survivors (Foa et al., 1991; Cassiday, McNally \& Zeitlin, 1992) and ferry disaster survivors (Dalgleish \& Yule, 1993). In a study of adult survivors of childhood sexual abuse with current PTSD, some of whom had been revictimized later in life, Field and colleagues (2001) found that revictimized participants demonstrated longer response latencies toward sexual/victimization words than did those who had not been revictimized, providing possible evidence that repeated trauma exposure may be related to an increase in attentional bias. No non-maltreated controls were included in this study, however, which precluded examination of effects that could be specific to childhood maltreatment.

The dot probe or visual probe task (Mogg \& Bradley, 1999) is another experimental paradigm that allows measurement of attentional bias. In each trial of a typical dot probe task, a pair of stimuli, one neutral and one emotionally salient (e.g., threatening), appears on a computer 
screen for a brief duration (generally 500 to 1500 milliseconds). Upon the offset of these images, a probe (an asterisk or set of dots) replaces one image. The viewer must quickly press a button that corresponds to the position of the probe on the screen (left versus right). Faster responses to probes that replace emotionally salient stimuli are thought to reflect biases in visual attention toward emotional cues; faster responses to probes that follow neutral stimuli reflect biases away from emotionally-valenced cues (Bryant \& Harvey, 1997).

The dot probe task offers some advantages over the Stroop task in measuring attention biases in traumatized individuals. Unlike the Stroop, the dot probe task does not rely on interference to measure bias in attention allocation and thus provides a more direct measure of visual attention (Mogg \& Bradley, 1998). The dot probe also allows for examination of the direction of attention biases (either toward or away from threat). Pictures (such as those of facial expressions) can be used as stimuli in the dot probe paradigm, eliminating the effortful semantic processing that the Stroop task typically requires.

The use of pictorial stimuli, such as human facial expressions, also has the advantage of providing a potentially more ecologically valid method of measuring attention bias in individuals who have suffered interpersonal trauma. Human facial expressions are particularly salient signals in human communication (Ohman, 2002), and pictures of threat-related facial expressions have been found to consistently disrupt attentional processing in different populations of individuals with PTSD (Felmingham, Bryant \& Gordon, 2003; Rauch et al., 2000; Shin et al., 2005). For example, traumatized participants with and without PTSD were asked in one study to attend to threat-related facial expressions during functional neuroimaging. Results yielded evidence of abnormal patterns of neural activity during attention to these faces in different groups of individuals with PTSD, as compared to traumatized controls without PTSD (Shin et al., 2005). 
As such, the dot probe appears to provide a more precise, directional measure of bias in visual attention than the Stroop, with the further advantage that it can be modified to include stimuli that are both ecologically valid and salient for different types of traumatized populations.

Researchers have administered variants of the dot probe task to individuals with PTSD in four published studies. In one study, Bryant and Harvey (1997) presented word pairs (one neutral/one threat-related or one neutral/one positive) to adult survivors of motor vehicle accidents with PTSD diagnoses, subclinical PTSD symptoms, and trauma controls with low levels of anxiety. They found that subjects with PTSD diagnoses responded more quickly to probes that replaced words related to driving threat than to probes that replaced positive or neutral words, suggesting a bias to attend preferentially to threat cues; this attentional bias was not found in the other two groups (Bryant \& Harvey 1997). In another study, Dalgleish and colleagues (2003) found that children and adolescents with Generalized Anxiety Disorder or PTSD (combined into one group) demonstrated a comparable bias toward threat-related words, which was not observed in youth with depression or healthy controls.

Elsesser, Sartory, and Tackenberg (2004, 2005) also administered variations of the dot probe to trauma survivors and healthy controls in two recent studies. In the first study, they found that trauma survivors with Acute Stress Disorder (ASD), compared to healthy controls, demonstrated a tendency to direct their attention away from trauma-related pictures. Participants with chronic PTSD, in contrast, tended to direct their attention toward trauma-related pictures. Differences among mean attention bias scores for these three groups, however, were not statistically significant (Elsesser, Sartory \& Tackenberg, 2004). In their 2005 study, the authors administered a dot probe task to healthy controls and recent trauma survivors at two time points (time one for traumatized participants was shortly after the trauma occurred; for both groups, 
time two occurred three months after initial testing). They found that recent trauma survivors tended to direct their attention away from threat cues at time one and toward threat cues at time two, while healthy controls attended preferentially to threat cues at time one and away from threat cues at time two. Both studies demonstrate that patterns of visual attention allocation differ between trauma survivors and controls; further, among trauma survivors, the recency of the traumatic event may influence the direction of attentional bias.

These studies highlight the possibility that proximal, versus distal (particularly early life), trauma may relate differently to patterns of information-processing. Early life trauma appears to predict marked and enduring changes in information-processing styles, as well as behavioral responses and physiology (Heim et al., 1997; Repetti, Taylor \& Seeman, 2002; Salmon \& Bryant, 2002; Cicchetti \& Curtis, 2006). A number of studies suggest that processing of emotional cues, such as facial expressions, occurs atypically in maltreated youth (e.g., Pollak, Cicchetti \& Klorman,1998; Pollak et al., 2000); this may reflect one way in which children adapt to unpredictable and frightening environments (Pollak, 2003). While these studies have examined responses to emotional cues in children with histories of maltreatment, few have focused on attentional biases or have characterized participants in terms of psychopathology. To date, only one published study has examined attentional bias for facial expressions in children with maltreatment histories. Pine and colleagues (2005) found that maltreated children (most of whom were diagnosed with PTSD), unlike non-maltreated controls, demonstrated an attention bias away from threatening faces on the dot probe paradigm; small group size, however, prevented comparisons between maltreated children with and without PTSD.

A recent study used the dot probe to examine attention biases in young adults who reported histories of childhood maltreatment (Gibb, Schofield, \& Coles, 2009). The authors 
found significant differences in attention bias between participants who self-reported as maltreated and as non-maltreated; the maltreated group demonstrated attentional biases toward threat, a pattern that was not found in the non-maltreated group. These findings led the authors to speculate that childhood abuse may have an enduring effect on attentional biases for facial displays of emotion. However, the authors did not provide data regarding later-life trauma or current PTSD within their sample, which leaves open the question of the specificity of effects to childhood versus lifetime traumatic experiences and associated psychopathology.

In sum, research findings from two attention paradigms - the modified Stroop and the dot probe- have revealed evidence for attention biases in maltreated children, adults with maltreatment histories, and individuals with PTSD. While distinct lines of research indicate biased information processing in adults with PTSD and in maltreated children, no published studies to date have explicitly examined relationships among childhood maltreatment, adult PTSD, and patterns of information processing, particularly attentional biases. Given that only some individuals who are traumatized during childhood develop disorders such as PTSD, clarification of characteristics, such as patterns of attention that distinguish them from other traumatized peers, may provide a first step toward elucidating factors associated with increased vulnerability to post-traumatic psychopathology.

The proposed study was therefore designed to examine performance on an ecologically valid measure of attentional bias in a heterogeneous sample of adults with a) varying histories of childhood maltreatment, and b) with and without current PTSD symptoms. More specifically, the goal of this study was to examine associations among attention bias (either for threatening or positive cues), childhood maltreatment, and current PTSD symptomatology. Given that more proximal adverse events (trauma experienced in adulthood) are likely to influence attention bias 
scores, a secondary aim of this study was to examine the unique effects of childhood maltreatment on attentional biases after statistically controlling for variance associated with trauma experienced in adulthood. We hypothesized that attention bias for emotional cues would significantly mediate associations between childhood maltreatment and adult PTSD symptoms, and that incidence of childhood maltreatment would explain significantly more variance in attention bias scores, as compared to incidence of adult trauma.

\section{Methods}

\section{Participants}

Participants were recruited through an ongoing NIMH funded study of risk factors for PTSD in a highly traumatized, low socioeconomic status, urban population in the southeastern United States (see Bradley et al., 2008 and Binder et al., 2008 for details of study). Participants were recruited from the general medical clinics of a publicly funded, not-for-profit healthcare system that serves economically disadvantaged individuals. Patients attending these clinics have been found to exhibit high rates of childhood maltreatment and post-traumatic symptoms that vary considerably in severity (Binder et al., 2008; Bradley et al., 2008). Study procedures were approved by the institutional review boards of Emory University School of Medicine and Georgia State University, Atlanta, Georgia.

Patients were deemed eligible for participation if they were able to give informed consent and understand English, as determined by a study researcher. A total of 161 adult males and females aged 18-60 years participated in this study. Data from 32 of these participants, however, were excluded from analyses due to poor task performance (i.e., they skipped or made errors on more than $20 \%$ of trials), yielding a final sample of 129 participants. 
Participants in the final sample were primarily female (72\%; n=92) and AfricanAmerican (89.9\%; $n=116)$ and were, on average, 39.5 years of age ( $\mathrm{SD}=12.5)$. Most participants had obtained 12 years or fewer of education (60.5\%; n=78) and reported household monthly incomes of less than $\$ 1000$ (70.2\%; $n=87)$. On average, participants reported mild to moderate current depressive symptoms according to the Beck Depression Inventory (BDI; Beck, 1996). No significant differences in mean BDI score, years of education, and monthly income were found between participants who were or were not excluded from the final analysis based on task performance ( $\mathrm{p}>$.05). On average, participants reported mild PTSD symptoms, according to their PSS total scores (Mean PSS total score=13.63;12.33); many participants reported experiencing symptoms for one year or longer (48\%; $n=54)$. Demographic and clinical characteristics of the final sample are described in Table 1.

Measures

Dot Probe Task (Mogg \& Bradley, 1999). The dot probe is a computerized task that requires participants to respond rapidly to onscreen cues in the context of distracting information that is either emotional or neutral in nature. During each trial of the task, a pair of face photographs (both of the same actor) was presented for 500 milliseconds (ms). After the offset of the face pair, an asterisk was presented in the location that one of the faces had occupied. Participants indicated as quickly as possible, with a forced-choice button press response, whether the asterisk appeared on the left- or right-hand side of the screen. In most face pairs, one face displayed an emotional expression (either threatening or happy) and the other a neutral expression; control trials with two neutral faces were also included. The task consisted of 80 trials (32 positive-neutral face pairs, 32 neutral-threat face pairs, and 16 neutral-neutral face pairs) presented in random order. During emotion pair trials, the probe replaced emotionally- 
valenced stimuli during half of the trials, and replaced neutral stimuli during the other half of the trials. The probe appeared on the left or right side of the screen an equal number of times. During half of the neutral-neutral trials, the probe appeared on the left and during the other half, it appeared on the right.

Emotion bias scores were calculated based on responses to threat-neutral and happyneutral pair trials. Separately for each type of pair (threat, happy), mean response time to emotion-congruent stimuli (probes that replace happy or threatening pictures was subtracted from) response time to emotion-incongruent stimuli (probes that replace neutral pictures), yielding threat bias and happy bias scores. Positive scores indicate a bias to direct attention toward emotional cues; negative scores indicate a bias to direct attention away from emotional cues. Although this task has been widely used in experimental settings, no published data regarding reliability are available. Findings from prior research, however, suggest that the measure validly discriminates between anxious and non-anxious adults and youth (Bradley, Mogg et al. 1999; Wilson and MacLeod 2003; Mogg, Philippot et al. 2004; Pine, Mogg et al. 2005).

PSS. The Modified PTSD Symptom Scale (PSS; Falsetti, Resnick et al. 1993), a brief self-report questionnaire with demonstrated diagnostic validity, provided a measure of presence and severity of PTSD symptomatology. The PSS was administered orally by trained clinicians to ensure that participants understood all items. The PSS assesses re-experiencing, avoidance, and arousal symptoms that occurred in the 2 weeks prior to test administration. Participants were asked to rate frequency of 17 such symptoms using a Likert-type scale. Frequency ratings range from 0 (not at all) to 3 (5 or more times per week/very much/almost always); severity ratings for 
endorsed items range from 0 (not at all distressing) to 4 (extremely distressing). A final question assesses how long symptoms have been present ( $<1$ month to $>1$ year).

Consistent with prior literature, we summed the PSS frequency items to obtain a continuous measure of PTSD symptom severity ranging from 0 to 51. For this sample, the PSS frequency items had standardized Cronbach's $\alpha=.9$ (mean .78). PSS scores can be classified as either dichotomous or continuous variables. Only continuously classified scores were used in the present study. The PSS has good concurrent validity with the PTSD module of the structured clinical interview for DSM-III-R (Falsetti et al., 1993). The PSS also has adequate reliability; Foa and colleagues (1993) reported a Cronbach’s $\alpha$ of .91 for the total scale and a 1-month retest reliability of .74 (Foa et al., 1993).

CTQ. The Childhood Trauma Questionnaire (CTQ) is a 28-item self-report questionnaire that has shown acceptable reliability and validity in both clinical and community populations (Bernstein et al., 2003) as well as within the current population (Binder et al., 2008; Bradley et al., 2008). Bernstein and colleagues (2003) found moderate levels of agreement between therapist observation ratings and CTQ scores (as high as .59 for physical abuse) and good internal consistency scores (physical abuse $=0.83$ to 0.86 , emotional abuse $=0.84$ to 0.89 , and sexual abuse $=0.92$ to 0.95 ). The CTQ has also shown adequate convergent validity in that its indices significantly correlate with scores on the Childhood Trauma Interview (Bernstein et al., 1994), which is another measure of childhood trauma. The CTQ was administered orally by trained clinicians. The CTQ retrospectively measures frequency of childhood traumatic incidents classified into five categories: physical abuse, sexual abuse, emotional abuse, physical neglect, and emotional neglect. Trauma frequency ratings are made on a 5-point Likert scale: never true, rarely true, sometimes true, often true, and always true. As such, childhood maltreatment 
frequency was measured continuously, yielding four indices used for the purposes of this study: total childhood maltreatment (sum of all indices; values for missing data calculated with mean substitution), total sexual abuse, total physical abuse, and total emotional abuse. Neglect indices were excluded from the present study based on evidence that maltreatment and neglect differentially predict biases or errors in processing of emotional cues; maltreated, particularly physically abused, children have shown response biases to anger or threatening cues, while neglected children appear to have difficulties differentiating among expressions of emotion (Pollak et al., 2000). Thus, we chose to examine only maltreatment-related indices from the CTQ; total CTQ score served as the primary variable of interest in mediational analyses because we were examining frequency of maltreatment incidence, conceptualized broadly, within this sample rather than specific correlates of particular types of maltreatment.

TEI. The Traumatic Events Interview (TEI) is a clinician-administered questionnaire designed to assess number and type of traumatic incidents that a participant has experienced throughout his or her lifetime. The TEI includes 15 questions about a range of potential traumatic events, including “Have you experienced a sudden life-threatening illness?” and "Have you witnessed a family member or friend being attacked without a weapon?” For each event type, the TEI queries frequency of occurrence, age at onset of the "worst" incident, feelings of terror, horror, and helplessness (rated on a 0-2 severity scale) at worst incident, and subjective feelings that oneself or another person may die or be seriously injured at worst incident (rated on a 0-2 severity scale). The TEI was developed for the purposes of the Grady Trauma Project (under the auspices of which the present data were collected) and collection of reliability and validity data is underway (Gillespie et al., 2009). Only frequency estimates of trauma occurrence in childhood (childhood TEI) and adulthood (adult TEI) were used as variables in the present study. 


\section{Data Analysis}

Data were analyzed using Baron and Kenny’s (1986) multiple regression approach to assessing for the presence of mediator effects (Baron \& Kenny, 1986). First, CTQ scores were examined as predictors of PTSD symptoms (as measured by the PSS) to establish that there was an association to mediate (Path C). Second, CTQ scores were examined as a predictor of attention bias (Path A). Once these paths were established, PSS score (PTSD symptoms) was regressed on CTQ scores after controlling for the effects of attention bias (Path B). If, after entering attention bias scores, an attenuated or insignificant relationship exists between childhood maltreatment and attention bias, then PTSD symptoms may be seen as a mediator of the relationship between childhood maltreatment and attention bias. As Baron and Kenny (1986) caution, full mediation, in which the effects of Path $\mathrm{C}$ are reduced to complete non-significance, is uncommon in psychological research. However, significant changes in regression coefficients when potential mediators are added to the model, as measured using the Sobel test, may provide a measure of the strength of the mediator (PTSD symptoms).

A second regression analysis focused on Path $\mathrm{C}$ was conducted to examine the unique contributions of childhood and adult trauma, measured by the TEI, toward attention bias scores. In this analysis, CTQ and TEI scores were entered as predictors of attention bias.

\section{Mediational Analyses}

\section{Results}

Correlations between attention bias scores and indices of the CTQ and PSS, as well as other clinical measures, are presented in Table 2. As predicted, all three types of childhood maltreatment (CTQ sexual, physical and emotional abuse subscale scores) correlated significantly and positively with the PSS indices for each of the three clusters of PTSD symptoms. CTQ total score was associated most strongly with PTSD hyperarousal symptoms ( $r$ 
$=.54, p<.01)$. While no statistically significant relationships were evident between childhood maltreatment and attention bias either toward or away from threatening faces, attention bias toward happy faces was significantly and positively associated with all childhood maltreatment types. The strongest association was found between happy bias scores and total incidence of childhood maltreatment (CTQ total score $(r=.25, p<.01)$.

According to Baron and Kenny (1986), mediational effects cannot be identified unless statistically significant associations exist among predictor and mediator variables (Path A), mediator and outcome variables (Path B), and predictor and outcome variables (Path C). For this study, we tested the statistical significance of associations between childhood maltreatment and attention bias (for threatening or happy faces, each examined separately) (Path A), attention bias and PTSD symptoms (Path B), and childhood maltreatment and PTSD symptoms (Path C). Statistically significant associations were found between attention bias for happy, but not threatening, faces and the other variables; therefore, mediational analyses were performed using only happy bias scores as the mediating variable (see Figure 1).

As shown in Figure 1, total reported frequency of childhood maltreatment predicted a significant amount of variance in happy bias scores (Path A; Beta=.25, R square=.06, $\mathrm{p}<.01$ ). Total incidence of childhood maltreatment also predicted $26.4 \%$ of the variance in total PTSD symptoms (Path C; Beta=.51, R square=.26, $\mathrm{p}<.01$ ). Attention bias for happy faces did not show a statistically significant association with total PTSD symptomatology $(\mathrm{r}=.15, n s)$. However, a statistically significant association was found between attention bias for happy faces and PTSD avoidance and numbing symptoms $(\mathrm{r}=.19, \mathrm{p}<.05)$. Happy bias thus accounted for a statistically significant proportion of variance in PTSD avoidance/numbing symptoms (Beta=.19, R square $=.04, \mathrm{p}<.05$ ). Given these findings, the Path $\mathrm{C}$ analysis was repeated to examine 
associations between childhood maltreatment and PTSD avoidance/numbing symptoms specifically; total incidence of maltreatment explained $20.8 \%$ of the variance in PTSD avoidance/numbing symptoms (Beta=.46, R square=.21, $\mathrm{p}<.01$; see Figure 1).

Sobel's test for indirect effects, which was used to test the statistical significance of attention bias as a mediating variable, yielded a value of $1.63(\mathrm{p}=.10)$ for the model in which PTSD avoidance and numbing symptoms served as the outcome variable. Thus, although attention bias toward happy faces was independently associated with both childhood maltreatment and PTSD avoidance and numbing symptoms, it did not significantly mediate the relationship between these two constructs.

Secondary Analysis

A secondary regression analysis was conducted to examine the unique contribution of childhood maltreatment to attention bias toward happy faces after accounting for the contributions of adult trauma. TEI total incidence of adult trauma score contributed a significant amount of variance to happy bias scores (Beta $=.22$, R square $=.05, \mathrm{p}<.05$ ); however, when added to this model, total incidence of childhood trauma accounted for more variance in happy bias scores than did incidence of adult trauma ( $\mathrm{R}$ square $=.09, \mathrm{p}<.01$ ). These results are detailed in Table 3.

\section{Discussion}

The findings from this study indicate a complex set of relationships among childhood maltreatment, early-stage information processing, and post-traumatic psychopathology in a sample of highly traumatized, economically disadvantaged adults, most of whom are members of an underserved racial minority group. We examined whether two types of attention bias (bias toward/away from threatening faces, bias toward/away from happy faces) mediated the 
association between childhood maltreatment and PTSD symptomatology. While neither type of attention bias was found to be a statistically significant mediator according to a conservative test of indirect effects (Sobel test; Baron \& Kenny, 1986), attention bias and childhood maltreatment demonstrated distinct associations with PTSD symptomatology, particularly avoidance and numbing symptoms, in adulthood. Additionally, a secondary analysis indicated that childhood maltreatment uniquely predicted a significant amount of variance in attention bias scores after controlling for the effects of traumatic experiences in adulthood.

Notably, adult survivors of childhood sexual, physical, or emotional abuse showed an attentional bias toward happy faces, relative to neutral faces. This pattern of findings is surprising, given that attention bias toward positive social cues has been shown more typically to relate to adaptive emotion regulation (Joorman \& Gotlib, 2007). One possible explanation for our findings is that participants in the present study have learned to attend to positive cues as a means of coping with constant environmental adversity. The majority of participants in this study sample were poor and had had limited access to educational opportunities. They also had more complex trauma histories than have participants in many studies in the PTSD information processing literature; this is illustrated in published studies on the population sampled for the present study (Binder et al., 2008; Bradley et al., 2008). Thus, selective attention for positive cues may be a necessary skill for survival in a challenging, frequently punitive, environment in which such positive feedback may be rare, particularly from racial majority group members, like those depicted in most of the study stimuli.

However, we found that this bias toward happy faces was also significantly associated with PTSD symptomatology, particularly avoidance and numbing. This suggests that, while an attentional preference for positive social cues be adaptive, it is possible that this selective 
attention for positive social cues could play a role in maintaining post-traumatic psychopathology. For example, rather than reflecting a preference for positive cues, overattention to overtly positive emotional cues may instead reflect a tendency to neglect or actively avoid other environmental cues, including both overtly threatening signals and emotionally ambiguous signals (such as a neutral facial expression), that may be misperceived as threatening (Lee et al., 2008). Avoidance of trauma-related cues (both physical reminders and thoughts of the trauma) contributes significantly to the maintenance of PTSD by preventing affected individuals from confronting feared trauma stimuli and thus precludes learning to extinguish exaggerated fear responses (Rothbaum \& Davis, 2003). In keeping with this conceptualization, and partially consistent with Pine et al.’s (2005) findings of threat avoidance in maltreated children with PTSD, the attention bias toward happy faces observed in our initial analyses may reflect avoidant tendencies rather than hyperattention to positive cues.

The present data extend earlier findings of attention biases on the Stroop task in veteran samples to an understudied population and could provide evidence of a threat-avoidant, rather than a threat-vigilant, attentional style in maltreated individuals with post-traumatic symptomatology. Notably, childhood maltreatment explained more variance in attentional biases than did adult trauma. Consistent with Gibb et al.'s (2009) findings, this could suggest that biased cognitive processing styles that develop early in life might confer more vulnerability for developing post-traumatic psychopathology after the experience of trauma as an adult. However, whether this attention bias represents a risk factor for PTSD or is part of PTSD sequelae remains unclear, due to the cross-sectional nature of this study.

Negative experiences that extend beyond severe trauma or biased face emotion discrimination may also have influenced the pattern of findings in the present sample. In 
particular, histories of racial discrimination may be important to consider in explaining the observed patterns of attentional response to the largely White/Caucasian stimulus faces in the dot probe task within our largely (90\%) African-American sample. Whether a more diverse set of faces or a primarily African-American stimulus set would have yielded a different pattern of results is not clear; further study of this question is warranted. More generally, participants may have interpreted "neutral” faces as negative, regardless of other stimulus characteristics, such as race. Impaired discrimination between threatening and neutral emotional signals has been observed previously in individuals with PTSD; Felmingham and colleagues (2003) found that individuals with PTSD demonstrated similar event-related potentials (ERPs) to angry and neutral faces, while non-traumatized controls had distinctly different ERPs to these stimuli, leading the authors to conclude that the PTSD group had difficulty differentiating these emotional signals. Interestingly, attention bias toward happy faces was not associated with intrusive and hyperarousal PTSD symptoms. It is possible that attentional bias toward happy cues is related to an emotionally avoidant style; individuals with these attentional tendencies may experience marked avoidance of physical reminders of trauma more frequently than cognitive intrusions and hypervigilance. Thus, individuals with this attentional bias may potentially benefit most from PTSD treatment that targets such avoidance, namely, exposure-based therapy.

Additionally, given that dot probe stimuli consisted largely of White faces, and that our study sample was primarily African-American, our results could reflect the influence of variables, such as participant or stimulus features, that extend beyond the target variables under study. Social cognitive studies indicate that race, and racial attitudes, can significantly influence performance on face processing tasks. Some cognitive studies have found an out-group homogeneity bias in face perception — that is, individuals tend to over-generalize features of 
individuals outside their own racial group and individuate features of in-group members (for a review, see Messick and Mackie, 1989). Racial attitudes also appear to influence face emotion categorization; Hugenberg and Bodenhausen (2003) found that White participants with higher implicit levels of prejudice perceived hostility in African-American faces longer than they did in White faces when presented with a morphed hostile to happy face expression continuum (Hugenberg \& Bodenhausen, 2003).

Attitudes toward individuals from another racial background have been shown to affect attentional responses on the dot probe task; Richeson and Trawalter (2008) administered the dot probe to a sample of White undergraduates, using both short (30ms) and long (450ms) stimulus presentation times and neutral versus happy facial expressions from African-American and White actors. They found that participants who were externally motivated to respond nonprejudicially toward African-Americans tended to orient toward neutral African-American faces during shorter stimulus presentations, and away from neutral African-American faces during longer presentations; no attention bias was found for happy faces as a whole (Richeson \& Trawalter, 2008). Presumably, the neutral African-American faces were perceived to be more threatening than happy faces when more time was available for visual processing.

Thus, it is possible that our findings reflect a similar effect, in which experiences as a function of membership in a minority group that commonly experiences discrimination, biased participants' categorization of emotional cues displayed on White faces, such that they perceived even neutral faces as threatening. Such a categorization bias could then have affected threat bias scores. Replication of the present study with a more diverse dot probe face stimulus set would be helpful to address potential confounding effects of viewing other-race faces and racial discrimination on attention bias, particularly threat bias. The attentional patterns we observed 
may be demographically mediated; it is possible that different attentional tendencies could emerge in a population with different demographic characteristics. Additionally, given that a preponderance of maltreated participants in this sample also experienced trauma in adulthood, we were unable to include an adequately sized maltreatment-only comparison group, which would have allowed more precise examination of the associations among attention bias, childhood maltreatment, and later life trauma.

Several study limitations are worth noting. Given the cross-sectional nature of this study and the use of retrospective reports, we could not make assertions about causality or time of onset for PTSD symptoms or attentional biases. Prospective, longitudinal studies are required to examine the temporal onset of attentional biases and PTSD symptomatology. Additionally, our meditational model suggested that attentional biases make a relatively small contribution to variance in PTSD symptoms. Although this is not surprising, given the complex trauma histories of many participants within this population, this illustrates that, relative to extrapersonal and environmental variables (such as trauma exposure and socio-economic status), cognitive/information-processing variables may play a small role in the development of PTSD.

The data reported here indicate that biases in early-stage information processing partially mediate the relationship between childhood maltreatment and some aspects of PTSD symptomatology in our highly traumatized and underprivileged sample. This is the first known study to examine associations among childhood maltreatment, attentional biases, and PTSD. Individuals who experienced more frequent maltreatment in childhood demonstrated more of an attentional bias toward happy faces and reported experiencing more PTSD avoidance and numbing symptoms. Although the cross-sectional design of this study precluded assumptions about causative factors, it is possible that individuals who experience maltreatment early in their 
development are more likely to develop a biased emotion processing style. If these biases persist into adulthood, they may prevent appropriate processing of all relevant environmental information; this could be a source of vulnerability toward the development of psychopathology in affected individuals, especially if they experienced multiple adverse events in adulthood. One recent study examined face emotion recognition in a sample of maltreated children with and without PTSD, as well as non-maltreated controls, and found that maltreated children, regardless of PTSD status, were faster to respond to emotional signals that conveyed threat (fearful faces) (Masten et al., 2008). This could suggest that maltreatment in and of itself is associated with risk for developing biases in emotion processing. Thus, increased public awareness, and early detection of, these attentional biases in survivors of early childhood maltreatment may increase the number of individuals obtaining appropriate treatments; this includes attentional retraining and other cognitively-based treatments that serve to correct attentional biases.

Given that we observed differences in type and direction of bias with a measure of attentional bias that is more direct, precise, and adaptable than the most widely-used measure of attentional bias (the Stroop) our findings indicate that precise, ecologically-valid measures are needed in PTSD information-processing research. Further studies of individuals with similar demographic characteristics are warranted to better differentiate information-processing mechanisms of psychopathology and resilience for this understudied population. The use of a more racially diverse dot probe face stimulus set and different stimulus onset times can address potential threats to ecological validity and better differentiate attentional patterns at different stages of processing. Concurrent collection of psychophysiological data, using techniques such as electroencephalography (EEG) or fMRI, could also provide evidence regarding potential biological mechanisms of these processes. 


\section{References}

Armony, J. L., Corbo, V., Clement, M. H., \& Brunet, A. (2005). Amygdala response in patients with acute PTSD to masked and unmasked emotional facial expressions. American Journal of Psychiatry, 162(10), 1961-1963.

Baron, R. M. \& Kenny, D.A. (1986). The moderator-mediator variable distinction in social psychological research: Conceptual, strategic, and statistical considerations. Journal of Personality and Social Psychology, 51(6), 1173-1182.

Beck, A. T., Steer, R. A., \& Brown, G. (1996). Manual for Beck Depression Inventory II (BDIII). San Antonio, Texas: Psychology Corporation.

Bernstein, D.P., Fink, L., Handelsman, L., Foote, J., Lovejoy, M., et al. (1994). Initial reliability and validity of a new retrospective measure of child abuse and neglect. American Journal of Psychiatry, 151(8), 1132-1136.

Bernstein, D. P., Stein, J. A., Newcomb, M. D., Walker, E., Pogge, D., Ahluvalia, T., et al. (2003). Development and validation of a brief screening version of the Childhood Trauma Questionnaire. Child Abuse and Neglect, 27, 169-190.

Binder, E. B., Bradley, R. G., Liu, W., Epstein, M. P., Deveau, T. C., Mercer, K. B., et al. (2008). Association of FKBP5 polymorphisms and childhood abuse with risk of posttraumatic stress disorder symptoms in adults. JAMA, 299(11), 1291-1305.

Bradley, B. P., Mogg, K., White, J., Groom, C., \& de Bono, J. (1999). Attentional bias for emotional faces in generalized anxiety disorder. British Journal of Clinical Psychology, 38, 267-278.

Bradley, R. G., Binder, E. B., Epstein, M. P., Tang, Y., Nair, H. P., Liu, W., et al. (2008). Influence of child abuse on adult depression: moderation by the corticotropinreleasing hormone receptor gene. Archives of General Psychiatry, 65(2), 190-200.

Bremner, J. D., Southwick, S.M., Johnson, D.R., Yehuda, R., Charney, D.S. (1993). Childhood physical abuse and combat-related posttraumatic stress disorder in Vietnam veterans. American Journal of Psychiatry, 150, 235-239.

Bryant, R. A. \& Harvey, A.G. (1997). Attentional bias in posttraumatic stress disorder. Journal of Traumatic Stress, 10, 635-644.

Buckley, T. C., Blanchard, E. B., \& Neill, W. T. (2000). Information processing and PTSD: A review of the empirical literature. Clinical Psychology Review, 28(8), 1041-1065.

Cassiday, K. L., McNally, R. J., \& Zeitlin, S. B. (1992). Cognitive processing of trauma cues in rape victims with post-traumatic stress disorder. Cognitive Therapy and Research, 16, 283-295. 
Chernick, M. R. (1999). Bootstrap Methods, A Practitioner's Guide.: Wiley Series in Probability and Statistics.

Cicchetti, D., \& Curtis, W. J. (2006). The developing brain and neural plasticity: Implications for normality, psychopathology, and resilience. In D. Cicchetti \& D. Cohen (Eds.), Developmental Psychopathology: Developmental Neuroscience (2nd ed., Vol 2, pp. 164). New York: Wiley.

Dalgleish, T., Taghavi, R., Hamid, N., Moradi, A., Canterbury, R., \& Yule, W. (2003). Patterns of processing bias for emotional information across clinical disorders: a comparison of attention, memory, and prospective cognition in children and adolescents with depression, generalized anxiety, and Posttraumatic Stress Disorder. Journal of Clinical Child and Adolescent Psychology, 32, 10-21.

Elsesser, K., Sartory, G., \& Tackenberg, A. (2004). Attention, heart rate, and startle response during exposure to trauma-relevant pictures: a comparison of recent trauma victims and patients with posttraumatic stress disorder. Journal of Abnormal Psychology, 113, 289301.

Elsesser, K., Sartory, G., \& Tackenberg, A. (2005). Initial symptoms and reactions to traumarelated stimuli and the development of posttraumatic stress disorder. Depression and Anxiety, 21, 61-70.

Falsetti, S. A., Resnick, H. S., Resnick, P. A., \& Kilpatrick, D. G. (1993). The modified PTSD symptom scale: A brief self-report measure of posttraumatic stress disorder. Behavior Therapy, 16, 161-162.

Felmingham, K. L., Bryant, R. A., \& Gordon, E. (2003). Processing angry and neutral faces in post-traumatic stress disorder: an event-related potentials study. Neuroreport, 14(5), 777780.

Field, N. P., Classen, C., Butler, L. D., Koopman, C., Zarcone, J., \& Spiegel, D. (2001). Revictimization and information processing in women survivors of childhood sexual abuse. Anxiety Disorders, 15, 459-469.

First, M. B., Spitzer, R. L., Williams, J. B. W., \& Gibbon, M. (1995). Structured Clinical Interview for DSM-IV. Washington, D.C.: American Psychiatric Press.

Foa, E. B., Feske, U., Murdock, T.B., Kozac, M.J. \& McCarthy, P.R. (1991). Processing of threat-related material in rape victims. Journal of Abnormal Psychology, 100, 156-162.

Foa, E. B. \& Kozak, M.J. (1986). Emotional processing of fear: Exposure to corrective information. Psychological Bulletin, 99, 20-35. 
Foa, E. B., Riggs, D. S., Dancu, C. V., \& Rothbaum, B. O. (1993). Reliability and validity of a brief instrument for assessing post-traumatic stress disorder. Journal of Traumatic Stress, 6, 459-473.

Gibb, B. E., Schofield, C. A., \& Coles, M. E. (2009). Reported history of childhood abuse and young adults' information-processing biases for facial displays of emotion. Child Maltreat, 14(2), 148-156.

Gillespie, C. F., Bradley, B., Mercer, K., Smith, A. K., Conneely, K., Gapen, M., et al. (2009). Trauma exposure and stress-related disorders in inner city primary care patients. Gen Hosp Psychiatry, 31(6), 505-514.

Golier, J. A., Yehuda, R., Bierer, L. M., Mitropoulou, V., New, A. S., Schmeidler, J., et al. (2003). The relationship of borderline personality disorder to posttraumatic stress disorder and traumatic events. American Journal of Psychiatry, 160, 2018-2024.

Heim, C., Owens, M. J., Plotsky, P. M., \& Nemeroff, C. B. (1997). Persistent changes in corticotropin-releasing factor systems due to early life stress: Relationship to the pathophysiology of major depression and post-traumatic stress disorder.

Psychopharmacology Bulletin, 33, 185-192.

Hugenberg, K., \& Bodenhausen, G. V. (2003). Facing Prejudice: Implicit prejudice and the perception of facial threat. Psychological Science, 14, 640-643.

Joormann, J., \& Gotlib, I. H. (2007). Selective attention to emotional faces following recovery from depression. Journal of Abnormal Psychology, 116(1), 80-85.

Kaspi, S. P., McNally, R. J., \& Amir, N. (1995). Cognitive processing of emotional information in posttraumatic stress disorder. Cognitive Therapy and Research, 19(4), 433-444.

Lee, E., Kang, J. I., Park, I. H., Kim, J. J., \& An, S. K. (2008). Is a neutral face really evaluated as being emotionally neutral? Psychiatry Research, 157, 77-85.

Masten, C. L., Guyer, A. E., Hodgdon, H. B., McClure, E. B., Charney, D. S., Ernst, M., et al. (2008). Facial expression recognition among maltreated children with high rates of Posttraumatic Stress Disorder. Child Abuse and Neglect, 32, 139-153.

Messick, D. M., \& Mackie, D. M. (1989). Intergroup relations. Annual Review of Psychology, 40, 45-81.

McNally, R. J., English, G. E., \& Lipke, H. J. (1993). Assessment of intrusive cognition in PTSD: Use of the modified Stroop paradigm. Journal of Traumatic Stress, 6, 33-41.

McNally, R. J., Kaspi, S.P, Reimann, B.C. \& Zeitlin, S.B. (1990). Selective processing of threat cues in post-traumatic stress disorder. Journal of Abnormal Psychology, 99, 398-402. 
Mogg, K. \& Bradley, B.P. (1998). A cognitive-motivational analysis of anxiety. Behaviour Research and Therapy, 36, 809-848.

Mogg, K. \& Bradley, B.P. (1999). Some methodological issues in assessing attentional biases for threatening faces in anxiety: A replication study using a modified version of the probe detection task. Behaviour Research and Therapy, 37, 595-604.

Mogg, K., Philippot, P., \& Bradley, B. P. (2004). Selective attention to angry faces in clinical social phobia. Journal of Abnormal Psychology, 113(1), 160-165.

Ohman, A. (2002). Automaticity and the amygdala: Nonconscious responses to emotional faces. Current Directions in Psychological Science, 11, 62-66.

Pine, D. S., Mogg, K., Bradley, B. P., Montgomery, L., Monk, C. S., McClure, E. B., et al. (2005). Attention bias to threat in maltreated children: Implications for vulnerability to stress-related psychopathology. American Journal of Psychiatry, 162, 291-296.

Pollak, S. D., Cicchetti, D., Hornung, K. \& Reed, A. (2000). Recognizing emotion in faces: Developmental effects of child abuse and neglect. Dev Psychol, 36, 679-688.

Pollak, S. D. (2003). Experience-dependent affective learning and risk for psychopathology in children. Annals of New York Academy of Science, 1008, 102-111.

Pollak, S. D., Cicchetti, D., \& Klorman, R. (1998). Stress, memory, and emotion: Developmental considerations from the study of child maltreatment. Development and Psychopathology, 10, 811-828.

Pollak, S. D., Cicchetti, D., Hornung, K. \& Reed, A. (2000). Recognizing emotion in faces: Developmental effects of child abuse and neglect. Journal of Developmental Psychology, 36, 679-688.

Rauch, S. L., Whalen, P.J., Shin, L.M., McInerney, S.C., Macklin, M.L., Lasko, N.B. et al. (2000). Exaggerated amygdala response to masked facial stimuli in posttraumatic stress disorder: a functional MRI study. Biological Psychiatry, 47, 769-776.

Repetti, R. L., Taylor, S.E., \& Seeman, T.E. (2002). Risky families: Family social environments and the mental and physical health of offspring. Psychological Bulletin, 128, 330-366.

Richeson, J. A., \& Trawalter, S. (2008). The threat of appearing prejudiced and race-based attentional biases. Psychological Science, 19, 98-102.

Rothbaum, B. O., \& Davis, M. (2003). Applying learning principles to the treatment of posttrauma reactions. Annals of the New York Academy of Sciences, 1008, 112-121.

Salmon, K., \& Bryant, R.A. (2002). Posttraumatic stress disorder in children: The influence of developmental factors. Clinical Psychology Review, 22, 163-188. 
Shin, L. M., Wright, C. I., Cannistraro, P. A., et al. (2005). A functional magnetic resonance imaging study of amygdala and medial prefrontal cortex responses to overtly presented fearful faces in posttraumatic stress disorder. Arch Gen Psychiatry, 62, 273-281.

U.S. Department of Health and Human Services (1996). Adverse childhood experiences study. Retrieved November 26, 2008 from http://www.cdc.gov/nccdphp/ace/index.htm.

Vasterling, J. J. and K. Brailey (2005). Neuropsychological findings in adults with PTSD. Neuropsychology of PTSD: Biological, Cognitive, and Clinical Perspectives. J. J. Vasterling and C. R. Brewin. New York, The Guilford Press.

Vrana, S. R., Roodman, A., \& Beckham, J. C. (1995). Selective processing of trauma-relevant words in posttraumatic stress disorder. Journal of Anxiety Disorders, 9, 515-530.

Widom, C. S. (1999). Posttraumatic stress disorder in abused and neglected children grown up. American Journal of Psychiatry, 156(8), 1223-1229.

Wilson, E. \& C. MacLeod (2003). Contrasting two accounts of anxiety-linked attentional bias: Selective attention to varying levels of stimulus threat intensity. Journal of Abnormal Psychology, 112(2), 212-218. 
Table I.

Demographics and Clinical Characteristics

\begin{tabular}{|c|c|c|c|}
\hline & Female & Male & Total \\
\hline Age mean (SD) & $38.61(12.47)$ & $40.9(12.41)$ & $39.5(12.51)$ \\
\hline \multicolumn{4}{|l|}{ Race } \\
\hline African American or Black & $82(89.1 \%)$ & $32(91.4 \%)$ & $116(89.9 \%)$ \\
\hline Caucasian or White & $7(7.6 \%)$ & $3(8.6 \%)$ & $10(7.8 \%)$ \\
\hline Other & $3(3.3 \%)$ & 0 & $3(2.4 \%)$ \\
\hline \multicolumn{4}{|l|}{ Education } \\
\hline$<12^{\text {th }}$ grade & $22(23.9 \%)$ & $6(17.1 \%)$ & $30(23.3 \%)$ \\
\hline $12^{\text {th }}$ grade or GED & $32(34.8 \%)$ & 16 (45.7\%) & $48(37.2 \%)$ \\
\hline some college or tech school & $22(23.9 \%)$ & $7(20.0 \%)$ & $29(22.5 \%)$ \\
\hline college or tech school graduate & $16(17.4 \%)$ & $5(14.3 \%)$ & $21(16.3 \%)$ \\
\hline graduate school & 0 & $1(2.9 \%)$ & $1(0.8 \%)$ \\
\hline \multicolumn{4}{|l|}{ Household monthly income } \\
\hline$\$ 0-\$ 249$ & 22 (25.3\%) & $11(31.4 \%)$ & $34(27.4 \%)$ \\
\hline$\$ 250-\$ 499$ & $9(10.3 \%)$ & $4(11.4 \%)$ & $13(10.5 \%)$ \\
\hline$\$ 500-\$ 999$ & $28(32.2 \%)$ & $12(34.3 \%)$ & $40(32.3 \%)$ \\
\hline$\$ 1000-\$ 1999$ & $19(21.8 \%)$ & $4(11.4 \%)$ & $24(19.4 \%)$ \\
\hline$\$ 2000+$ & $9(10.3 \%)$ & $4(11.4 \%)$ & $13(10.5 \%)$ \\
\hline CTQ total score - Mean (SD) & $43.99(22.81)$ & $40.80(18.02)$ & $42.94(21.41)$ \\
\hline Sexual abuse & $8.44(5.91)$ & $7.30(4.70)$ & $8.03(5.5)$ \\
\hline Physical abuse & $9.01(4.97)$ & $8.62(4.31)$ & $8.87(4.75)$ \\
\hline Emotional abuse & $9.98(6.05)$ & $8.70(4.62)$ & $9.66(5.63)$ \\
\hline PSS total-Mean (SD) & $13.01(12.16)$ & $14.97(12.88)$ & $13.63(12.33)$ \\
\hline Intrusive symptoms & $3.18(3.77)$ & 3.47 (3.61) & $3.27(3.71)$ \\
\hline Avoidance and Numbing & $5.40(5.64)$ & $7.16(6.22)$ & $5.94(5.83)$ \\
\hline Hyperarousal & $4.34(4.14)$ & $4.34(4.11)$ & $4.36(4.10)$ \\
\hline
\end{tabular}


PSS -Duration of symptoms

Less than 1 month

1-3 months

3 months - 1 year

$>1$ year
27 (24\%)

$8(7 \%)$

$10(9 \%)$

$38(34 \%)$
$9(8 \%)$

$2(2 \%)$

$3(3 \%)$

$16(14 \%)$
$36(31.9 \%)$

$10(9 \%)$

$13(12 \%)$

$54(48 \%)$ 
Table II.

Intercorrelations Among Attention Bias Scores and Clinical Measures

\begin{tabular}{llllllllll}
\hline 2 & 3 & 4 & 5 & 6 & 7 & 8 & 9 & 10
\end{tabular}

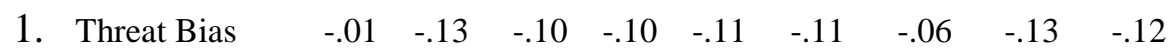

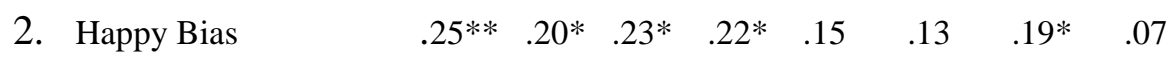

3. CTQ Total $\quad .78^{*} \quad .88^{* *} \quad .93^{* *} \quad .51^{* *} \quad .40^{* *} \quad .46^{* *} \quad .54^{* *}$

4. CTQ Sexual Abuse $\quad .59^{* *} .66^{* *} .37^{* *} \quad .27^{* *} \quad .32^{* *} \quad .42^{* *}$

5. CTQ Physical Abuse $\quad .81^{* *} .49^{* *} \quad .44^{* *} .42^{* *} \quad .48^{* *}$

6. CTQ Emotional Abuse $\quad .52^{* *} \quad .41^{* *} .46^{* *} \quad .56^{* *}$

$\begin{array}{llll}\text { 7. PSS Total } & .85^{* *} & .94^{* *} & .89^{* *}\end{array}$

8. PSS Intrusive Symptoms $\quad .69^{* *} .66^{* *}$

9. PSS Avoidance/Numbing $\quad .75^{* *}$

10. PSS Hyperarousal

$* \mathrm{p}<.05$

$* * \mathrm{p}<.01$ 
